Pacific Journal of Mathematics

FOURIER COEFFICIENTS OF THE RUDIN-CARLESON 


\section{FOURIER COEFFICIENTS OF THE RUDIN-CARLESON EXTENSIONS}

\section{J. GLobeVNIK}

If $F$ is a closed subset of the unit circle in $C$ of Lebesgue measure 0 then by the Rudin-Carleson theorem every continuous function $f: F \rightarrow C$ has a norm-preserving extension belonging to the disc algebra. If we prescribe some Fourier coefficients of the extension $g$ then in general the norm of $g$ will exceed the norm of $f$. In the paper we relate this problem to an extremal problem and give optimal estimations of the norms of extensions with prescribed Fourier coefficients. In particular, we give a precise description of those functions $f$ which have normpreserving extensions $g$ with finitely many prescribed Fourier coefficients.

Introduction. Denote by $C(K)$ the Banach space of all continuous complex-valued functions on a compact Hausdorff space $K$, with sup norm, and let $T$ be the unit circle in $C$. Given $f \in C(T)$ we denote the Fourier coefficients of $f$ by $\hat{f}(n)$, i.e.,

$$
\hat{f}(n)=\frac{1}{2 \pi} \int_{-\pi}^{\pi} e^{-i n \theta} f\left(e^{i \theta}\right) d \theta \quad(n=0, \pm 1, \pm 2, \cdots) .
$$

We write $A$ for the disc algebra, i.e., the closed subspace of $C(T)$ of those functions which have continuous extensions to the closed unit disc, analytic in its interior, or equivalently, of those functions in $C(T)$ whose negative Fourier coefficients vanish [12].

Denote by $N$ the set of all nonnegative integers and let $F \subset T$ be a closed set of Lebesgue measure 0 . By the well-known RudinCarleson theorem [12,17] every $f \in C(F)$ has an extension $g \in A$. It follows easily that given $n \in N$ and complex numbers $a_{i}(0 \leqq i \leqq n)$ there always exists an extension $h \in A$ of $f$ which satisfies $\hat{h}(i)=$ $a_{i}(0 \leqq i \leqq n)$. More generally, there are infinite sets $E \subset N$ and complex-valued functions $a$ on $E$ such that given any closed set $F \subset T$ of Lebesgue measure 0 every $f \in C(F)$ has an extension $h \in A$ satisfying

$$
\hat{h}(n)=a(n) \quad(n \in E)
$$

[see 18].

In the Rudin-Carleson theorem the extension $g$ of $f$ can be chosen so that $\|g\|=\|f\|$. Clearly this is no longer possible in 
general if one prescribes the Fourier coefficients of the extension as in (2) so it is natural to ask: Which functions $f \in C(F)$ admit normpreserving extensions $h \in A$ satisfying (2)? If a function $f \in C(F)$ has no such extensions what can be said about the norms of its extensions $h \in A$ satisfying (2)?

We will see that for a large class of sets $E$ the answer is extremely simple and is related to an extremal problem: Let $M_{0}=$ inf $\|h\|$ where inf is taken over all $h \in A$ satisfying (2). Then for arbitrary $M>M_{0}$ every $f \in C(F)$ has an extension $h \in A$ satisfying $\|h\|=\max \{\|f\|, M\}$. In all cases considered here the set of extremal functions (i.e., functions $h \in A$ satisfying (2) and $\|h\|=M_{0}$ ) is too small to allow extremal interpolation (i.e., interpolation in the case $M=M_{0} \neq 0$ ).

Section 1 contains the main results about the extensions with prescribed Fourier coefficients. In $\S 2$ we first prove the crucial Lemma 2.2 which enables us to extend [10, Theorem] to subspaces of the disc algebra. This theorem is our main tool which we then use to prove the results from $\S 1$. In $\S 3$ we consider first some special cases and then we show that our main tool can be applied to prove some other interpolation theorems.

We denote by $\triangle, \bar{J}, T$ the open unit disc in $C$, its closure and its boundary, respectively. Let $K$ be a compact Hausdorff space and let $X$ be a complex Banach space. By $C(K, X)$ we denote the Banach space of all continuous functions from $K$ to $X$, with sup norm, and write $C(K)$ for $C(K, C)$. The Fourier coefficients of $f \in$ $C(T, X)$ are defined by (1) (the integral being the Riemann integral [11]). We denote by $A(X)$ the closed subspace of $C(T, X)$ of those functions which admit continuous extensions to $\bar{J}$, analytic [11] on $\Delta$, or equivalently, of those functions whose negative Fourier coeffcients vanish [15] and write $A$ for $A(C)$. Sometimes we identify the functions in $A(X)$ with their analytic extensions. If $B$ is a subspace of $C(K)$ we denote by $B \otimes X$ the closed span in $C(K, X)$ of functions of the form $z \rightarrow \phi(z) x(\phi \in B, x \in X)$. We denote by $M(K)$ the space of complex regular Borel measures on $K$ of bounded variation; if $\mu \in M(K)$ we denote its total variation by $|\mu|$ and if $F \subset K$ is a closed set we define $\mu_{F} \in M(K)$ by $\mu_{F}(U)=\mu(U \cap F)$ ( $U$ Borel set in $K$ ). If $B$ is a subspace of $C(K)$ we denote by $B^{\perp}$ its annihilator in $M(K)$. We write $\bar{S}$ for the closure of a set $S \subset X$ and denote by $B_{r}(X)$ the open ball in $X$ of radius $r$, centered at the origin. We say that a map $\Phi: K \rightarrow 2^{X}$ (where $2^{X}$ is the set of all subsets of $X)$ is open [10] if the set $\{(s, x): s \in K, x \in \Phi(s)\}$ is open in $K \times X$. If $\left\{S_{\alpha} ; \alpha \in A\right.$ is a family of open subsets of $X$ with $x_{\alpha} \in$ $\bar{S}_{\alpha}\{\alpha \in A)$ we say that $S_{\alpha}$ are equilocally connected at $x_{\alpha}$ if given 
any $\varepsilon>0$ there is some $\delta>0$ such that $S_{\alpha} \cap\left(x_{\alpha}+B_{\delta}(X)\right)$ lies in a conected component of $S_{\alpha} \cap\left(x_{\alpha}+B_{\varepsilon}(X)\right)$ for each $\alpha \in A$. We denote by $N$ the set of all nonnegative integers.

1. Main results. Call a closed subspace $B$ of $C(T)$ a Riesz subspace if every $\mu \in B^{\perp}$ is absolutely continuous (with respect to Lebesgue measure on $T$ ). By F. and M. Riesz theorem [12] $A$ is a Riesz subspace of $C(T)$. There are proper closed subspaces of $A$ having this property. Let $E \subset N$ and denote

$$
A_{E}=\{f \in A: \hat{f}(n)=0(n \in E)\} .
$$

If $E$ is finite then it is easy to see that $A_{E}$ is a Riesz subspace of $C(T)$. Rudin [14] observed that the same is true for a large class of infinite sets $E$. We call a set $E \subset N$ a Rudin set if $A_{E}$ is a Riesz subspace of $C(T)$. At present no characterization of Rudin sets seems to be known although there are many examples in the literature $[4,5,14]$ the simplest being the Hadamard gap sequences [14].

Denote by $m$ the Lebesgue measure on $T$ and let $V$ be a subspace of $M(T)$. We say that $m$ is $V$-absolutely continuous if $\mu \in$ $V-\{0\},|\mu|(S)=0$ implies that $m(S)=0$. Note that this definition is different from the one used in [8]. By F. and M. Riesz theorem $A^{\perp}$ can be identified by $H_{0}^{1}$, the space of functions in $L^{1}(T)$ whose nonpositive Fourier coefficients vanish [12]. Since no function in $H_{0}^{1}$ can vanish on a set of positive Lebesgue measure without vanishing a.e. [12] it follows that $m$ is $A^{\perp}$-absolutely continuous and consequently $m$ is $A_{E}^{\perp}$-absolutely continuous for any finite set $E \subset N$.

THEOREM 1.1. Let $X$ be a complex Banach space and let $E \subset N$ be a Rudin set. Assume that $a: E \rightarrow X$ is a function such that

$$
\hat{g}(n)=a(n) \quad(n \in E)
$$

for some $g \in A(X)$. Put

$$
M_{0}=\inf \{\|g\|: g \in A(X), \hat{g}(n)=a(n) \quad(n \in E)\}
$$

and let $F \subset T$ be a nonempty closed set of Lebesgue measure 0 . If $M>M_{0}$ then given any $f \in C(F, X),\|f\| \leqq M$ there is some $g \in A(X)$ (4) which extends $f$ and satisfies (3) and $\|g\| \leqq M$.

Suppose, in addition, that the Lebesgue measure on $T$ is $A_{E^{-}}^{\perp}$ absolutely continuous. Then any function $g \in A(X)$ satisfying (3) and $\|g\|=M_{0}$ satisfies $\|g(t)\| \equiv\|g\|(t \in T)$ and consequently (4) is false for $M=M_{0}$ if $M_{0} \neq 0$. 
The following corollary gives a complete answer to the questions in Section 0 in the case when $E$ is finite.

Corollary 1.2. Let $X$ be a complex Banach space, $E \subset N$ a finite set and $a: E \rightarrow X$ a function. Put $M_{0}=\inf \{\|g\|: g \in A(X)$, $\hat{g}(n)=a(n)(n \in E)\}$ and let $F \subset T$ be a nonempty closed set of Lebesgue measure 0. Assume that $M>M_{0}$. Then given any $f \in$ $C(F, X),\|f\| \leqq M$ there is an extension $g \in A(X)$ of $f$ which satisfies $\|g\| \leqq M$ and $\hat{g}(n)=a(n) \quad(n \in E)$. This is false for $M=M_{0}$ if $M_{0} \neq 0$.

\section{Proofs.}

LEMMA 2.1. $[1,16]$. Let $K$ be a compact Hausdorff space and let $F \subset K$ be a closed set. Let $B$ be a closed subspace of $C(K)$ with the property that $\mu \in B^{\perp}$ implies that $\mu_{F}=0$. Then given any positive continuous function $p$ on $K$ and any $f \in C(F)$ such that $\mid f(s \mid<p(s)$ $(s \in F)$ there exists $\tilde{f} \in B$ which extends $f$ and satisfies $|\widetilde{f}(z)|<p(z)$ $(z \in K)$.

LEMMA 2.2. Let $K, F$ and $B$ satisfy the assumptions of Lemma 2.1. Assume that $F=G \cup H$ where $G$ and $H$ are nonempty disjoint compact sets. Let $P$ be an open connected subset of a complex Banach space $X$ which contains the point 0 and let $x \in P$. Given any $\varepsilon>0$ and any neighborhood $U$ of $G$ there exists a function $f \in B \otimes X$ such that
(i) $f(K) \subset P$
(ii) $f(H)=\{0\}$
(iii) $f(G)=\{x\}$
(iv) $\|f(z)\|<\varepsilon(z \notin U)$.

Proof. Let $\varepsilon>0$ and let $U$ be a neighborhood of $G$. Since $P$ is open and connected there are $n \in N, \delta: 0<\delta<\varepsilon$ and $x_{i} \in P(0 \leqq$ $i \leqq n), x_{0}=0, x_{n}=x$ such that

$$
\max _{i}\left\|x_{i}-x_{i-1}\right\|<\delta
$$

and

$$
x_{i}+B_{3 \delta}(X) \subset P \quad(0 \leqq i \leqq n) .
$$

Assume that there are functions $f_{i} \in B \otimes X(1 \leqq i \leqq n)$ and neighborhoods $U_{i}(1 \leqq i \leqq n)$ of $G, U_{1}=U, U_{i} \subset U_{i-1}(2 \leqq i \leqq n)$ such that
(a) $\left\|f_{i}\right\|<2 \delta$
(b) $f_{i}(H)=\{0\}$ \}$(1 \leqq i \leqq n)$
(c) $f_{i}(G)=\left\{x_{i}-x_{i-1}\right\}$ 
(d) $\quad\left\|\sum_{k=1}^{i} f_{k}(z)-x_{i}\right\|<\delta / n\left(z \in U_{i+1} ; 1 \leqq i \leqq n-1\right)$

(e) $\left\|f_{i}(z)\right\|<\delta / n\left(z \notin U_{i} ; 1 \leqq i \leqq n\right)$.

Define $f=\sum_{i=1}^{n} f_{i}$. If $z \notin U$ then $z \notin U_{i}(1 \leqq i \leqq n)$ so by (e) $\|f(z)\|<$ $\delta$ which proves (iv). Also, for such $z, f(z) \in P$ by (6). For convenience, put $U_{n+1}=\phi$ and let $z \in U_{i}-U_{i+1}$ for some $i, 1 \leqq i \leqq n$. By (d) we have $\sum_{j=1}^{i-1} f_{j}(z) \in x_{i-1}+B_{\delta / n}(X)$. Since $z \notin U_{j}(i+1 \leqq j \leqq n)$ we have by (e) that $\sum_{j=i+1}^{n} f_{j}(z) \in B_{(n-i) \delta / n}(X)$. By (a) and by (6) it follows that $f(z) \in x_{i-1}+B_{\delta / n}(X)+f_{i}(z)+B_{(n-i) \delta / n}(x) \subset x_{i-1}+B_{3 \delta}(X) \subset P$ which proves (i). By (b) and (c), (ii) and (iii) are also satisfied.

It remains to prove the existence of $f_{i}$ and $U_{i}$. Put $U_{1}=U$. By Lemma 2.1 (using Urysohn lemma to get suitable $p$ ) there exists $\phi_{1} \in B$ such that $\phi_{1}(G)=\{1\}, \phi_{1}(H)=\{0\},\left\|\phi_{1}\right\|<2$ and $\left|\phi_{1}(z)\right|<1 / n(z \notin$ $\left.U_{1}\right)$. Define $f_{1}(z)=\phi_{1}(z)\left(x_{1}-x_{0}\right)(z \in K)$. Clearly $f_{1} \in B \otimes X$ and by (5), $f_{1}$ satisfies (a), (b), (c) and (e) above for $i=1$. Let $2 \leqq j \leqq n$ and assume that there are $f_{k} \in B \otimes X$ and $U_{k}(1 \leqq k \leqq j-1)$, neighborhoods of $G$, satisfying (a), (b), (c) and (e) for $1 \leqq i \leqq j-1$. By (c) $\left(\sum_{k=1}^{j-1} f_{k}\right)(G)=\left\{x_{j-1}\right\}$ so by the continuity of $f_{k}$ there exists $U_{j} \subset$ $U_{j-1}, U_{j}$ neighborhood of $G$, such that (d) is satisfied for $i=j-1$. Now again by Lemma 2.1 there is some $\dot{\phi}_{j} \in B$ such that defining $f_{j}(z)=\phi_{j}(z) \cdot\left(x_{j}-x_{j-1}\right), f_{j}$ satisfies (a), (b), (c) and (e) for $i=j$.

THEOREM 2.3. Let $K$ be a compact metric space and let $F \subset K$ be a closed set such that any finite cover of $F$ consisting of open balls has a finite refinement consisting of pairwise disjoint closed sets. Suppose that $B \subset C(K)$ is a closed subspace such that $\mu \in B^{\perp}$ implies that $\mu_{F}=0$.

Let $X$ be a complex Banach space and $\Phi: K \rightarrow 2^{X}$ an open map such that $g(z) \in \Phi(z)(z \in K)$ for some $g \in B \otimes X$. Let $f \in C(F, X)$ satisfy $f(s) \in \overline{\Phi(s)}(s \in F)$. Assume that the sets $\Phi(s)(s \in F)$ are connected and equilocally connected at the points $f(s)$. Then there is some $\tilde{f} \in B \otimes X$ which extends $f$ and satisfies $\widetilde{f}(z) \in \Phi(z)(z \in K-F)$.

Proof. Follow the proof of [10, Theorem] and use Lemma 2.2 instead of [9, Lemma 4].

Corollary 2.4. Let $K, F$ and $B$ satisfy the assumptions of Theorem 2.3 and let $X$ be a complex Banach space. Suppose that $g \in C(K, X)$ and let $M>0$. If there is some $h_{0} \in B \otimes X$ such that $\left\|g+h_{0}\right\|<M$ then given any $u \in C(F, X),\|u\| \leqq M$ there is an extension $\tilde{u}$ of $u$ of the form $\tilde{u}=g+h$ with $h \in B \otimes X$ such that $\|\widetilde{u}(z)\|<M(z \notin F)$.

Proof. Let $h_{0} \in B \otimes X$ satisfy $\left\|g+h_{0}\right\|<M$. Define $\Phi(z)=$ $\{x \in X:\|g(z)+x\|<M\}$ and $f(s)=u(s)-g(s)(s \in F)$. Clearly $h_{0}(z) \in$ 
$\Phi(z)(z \in K)$ and $f(s) \in \overline{\Phi(s)}(s \in F)$. By the continuity of $g$ and the convexity of $\Phi(s)(s \in F), \Phi$ and $f$ satisfy the assumptions of Theorem 2.3 and consequently there is some $\widetilde{f} \in B \otimes X$ which extends $f$ and satisfies $\widetilde{f}(z) \in \Phi(z)(z \notin F)$. Putting $\widetilde{u}=g+\widetilde{f}$ it is obvious that $\tilde{u}$ has the required properties.

Proof of Theorem 1.1. Let $M>M_{0}$ and let $f \in C(F, X),\|f\| \leqq M$. By definition of $M_{0}$ there is some $g_{0} \in A(X)$ such that $\left\|g_{0}\right\|<M$ and $\hat{g}_{0}(n)=a(n)(n \in E)$. By the assumption $A_{E}$ is a Riesz subspace of $C(T)$ so $F$ and $B=A_{E}$ satisfy the assumptions of Corollary 2.4. By Corollary 2.4 (with $h_{0}=0$ ) there is an extension $g$ of $f$ of the form $g=g_{0}+h$ with $h \in A_{E} \otimes X$ such that $\|g(z)\|<M(z \in T-F)$. Clearly $g \in A(X)$ and $\hat{g}(n)=a(n)(n \in E)$. This proves the first statement in Theorem 1.1.

REMARK. We see from the proof that under the assumptions of the first part of Theorem 1.1 one can prove (4) with $\|g\| \leqq M$ being replaced by $\|g(z)\|<M(z \in T-F)$.

The proof of Theorem 1.1 will be complete once we have proved the following

LeMma 2.5. Let $X$ be a complex Banach space and let $B \subset C(T)$ be a closed subspace such that the Lebesgue measure $m$ on $T$ is $B^{\perp_{-}}$ absolutely continuous. Assume that $L \subset C(T, X)$ is a closed subspace containing $B \otimes X$ and let $f \in C(T, X)$ and $g_{0} \in L$ satisfy

$$
\left\|f+g_{0}\right\|=\inf _{g \in L}\|f+g\| \text {. }
$$

Then

$$
\left\|f(t)+g_{0}(t)\right\| \equiv\left\|f+g_{0}\right\| \quad(t \in T) .
$$

Proof. Let $M\left(T, X^{\prime}\right)$ be the Banach space of all regular Borel measures $\mu$ with values in $X^{\prime}$ which have bounded variation $|\mu|$ with the norm $\|\mu\|=|\mu|(T)$. It is known [2,3] that $M\left(T, X^{\prime}\right)$ is isometric to $C(T, X)^{\prime}$ the pairing being $\langle\dot{\phi} \mid \mu\rangle=\int_{T} \phi d \mu(\phi \in C(T, X)$, $\mu \in M\left(T, X^{\prime}\right)$ ) (see [2,3] for the definition and the properties of the integral with respect to a vector measure). By the well-known duality relation $\left[6\right.$, p. 111] there is some $\mu \in L^{\perp},\|\mu\|=1$ such that

$$
\left\|f+g_{0}\right\|=\int_{T}\left(f+g_{0}\right) d \mu
$$

and it follows that 


$$
\left\|f+g_{0}\right\| \leqq \int_{T}\|f(t)+g(t)\| d|\mu| .
$$

Assume that (7) is false. By the continuity of $f$ and $g_{0}$ there is an $\varepsilon>0$ such that $\left\|f(t)+g_{0}(t)\right\|<\left\|f+g_{0}\right\|-\varepsilon(t \in U)$ on a nonempty open set $U \subset T$. Since $\|\mu\|=\int_{T} d|\mu|=1$ it follows by (8) that $|\mu|(U)=0$ and consequently $|\langle x \mid \mu(\cdot)\rangle|(U)=0(x \in X)$. Note that $m(U) \neq 0$. Since $\mu \in L^{\perp} \subset(B \otimes X)^{\perp}$ we have $\langle x \mid \mu(\cdot)\rangle \in B^{\perp}(x \in X)$. Now, by the assumption $m$ is $B^{\perp}$-absolutely continuous so it follows that $\langle x \mid \mu(\cdot)\rangle=0(x \in X)$ which implies that $\mu=0$, a contradiction.

3. Special cases and generalizations. Let $m \in N$. A function $f \in A$ of the form

$$
f(z)=e^{i \theta} \prod_{i=1}^{m} \frac{z-\alpha_{i}}{1-\bar{\alpha}_{i} z}(z \in \bar{J})
$$

$\left(f(z)=e^{i \theta}(z \in \bar{A})\right.$ if $\left.m=0\right)$ where $\theta \in \boldsymbol{R}$ and $\alpha_{i} \in \Delta(1 \leqq i \leqq m)$ is called a finite Blaschke product (of $m$ factors). Denote by $H^{\infty}$ the Banach space of all bounded complex-valued analytic functions $\Delta$, with sup norm.

If we prescribe the first $n$ Fourier coefficients of the scalarvalued Rudin-Carleson extension then the solution of the corresponding extremal problem is known:

Lemma 3.1. [6, pp. 139, 140, 143]. Let $a_{0}, a_{1}, \cdots, a_{n} \in C$. Among all functions $f$ of the form $f(z)=\sum_{i=0}^{n} a_{i} z^{i}+z^{n+1} g(z)(z \in \Delta)$ where $g \in H^{\infty}$ there is one with minimal norm. This function is unique and is a scalar multiple of a Blaschke product of $\leqq n$ factors. Its norm is equal to the greatest absolute value of the eigenvalues of the matrix

$$
\left[\begin{array}{ccccc}
a_{n} & a_{n-1} & \cdots & a_{1} & a_{0} \\
a_{n-1} & a_{n-2} & \cdots & a_{0} & 0 \\
\vdots & & & & \\
a_{0} & 0 & \cdots & \cdots & 0
\end{array}\right]
$$

Further, if $h(z)=\sum_{i=0}^{\infty} c_{i} z^{i}(z \in \Delta)$ is a scalar multiple of a Blaschke product of $\leqq n$ factors then $h$ is uniquely determined by $c_{0}, c_{1}, \cdots, c_{n}$.

REMARK. In particular, this shows that there is a unique function $f_{0} \in A$ such that $\left\|f_{0}\right\|=\inf \left\{\|f\|: f \in A, \hat{f}(i)=a_{i}(0 \leqq i \leqq n)\right\}$. Simple examples show that this is no longer true in the vector case although by Theorem 1.1 the extremal functions $f_{0}$ still have the property that $t \rightarrow\left\|f_{0}(t)\right\|$ is constant on $T$. 
Corollary 3.2. Let $M>0$ and let $a_{0}, a_{1}, \cdots, a_{n} \in C$. Suppose that $F \subset T$ is a nonempty closed set of Lebesgue measure 0 . Then the greatest absolute value of the eigenvalues of the matrix (9) is less than $M$ iff

$$
\begin{aligned}
& \text { given any } f \in C(F),\|f\| \leqq M \text { there is an extension } \\
& g \in A \text { of } f,\|g\| \leqq M \text { satisfying } \hat{g}(i)=a_{i}(0 \leqq i \leqq n)
\end{aligned}
$$

Our next corollary gives a surprisingly simple description of infinite sequences $a_{i}$ satisfying (10) for every $n$ :

Corollary 3.3. Let $M>0$ and let $\left\{a_{i}: i \in N\right\}$ be a sequence of complex numbers. Let $F \subset T$ be a nonempty closed set of Lebesgue measure 0 . The following are equivalent

(i) given any $n \in N$ and any $f \in C(F),\|f\| \leqq M$, there exists an extension $g \in A$ of $f,\|g\| \leqq M$, such that $\hat{g}(i)=a_{i}(0 \leqq i \leqq n)$.

(ii) the series $\sum_{i=0}^{\infty}(1 / M) a_{i} z^{i}$ converges on $\Delta$ to a function in $H^{\infty}$ whose norm does not exceed 1 and which is not a finite Blaschke product.

Proof. For $n \in N$ denote $p_{n}(z)=\sum_{i=0}^{n} a_{i} z^{i}$ and $A_{n}=\left\{u \in A: u / z^{n} \in\right.$ $A\}, H_{n}^{\infty}=\left\{u \in H^{\infty}: u / z^{n} \in H^{\infty}\right\}$. Note that by Lemma $3.1 \operatorname{dist}\left(p_{n}, A_{n+1}\right)=$ $\operatorname{dist}\left(p_{n}, H_{n+1}^{\infty}\right)(n \in N)$ and observe that by Lemma 3.1 and Corollary 3.2 (i) is equivalent to $\operatorname{dist}\left(p_{n}, A_{n+1}\right)<M(n \in N)$.

Let dist $\left(p_{n}, A_{n+1}\right)<M$ for all $n$. Then for some sequence $g_{n} \in$ $A_{n+1}$ we have $\left\|p_{n}+g_{n}\right\|<M(n \in N)$ so a subsequence of the sequence $p_{n}+g_{n}$ converges uniformly on compact subsets of $\Delta$ to a function $u \in H^{\infty},\|u\| \leqq M$ and consequently also the Fourier coefficients of $p_{n}+g_{n}$ converge to corresponding Fourier coefficients of $u$ so $u(z)=$ $\sum_{i=0}^{\infty} a_{i} z^{i}(z \in \Delta)$. Let $n \in N$ and suppose that $u / M$ is a Blaschke product of $\leqq n$ factors. Let $u_{0}=p_{n}+g, g \in H_{n+1}^{\infty}$ satisfy $\left\|u_{0}\right\|=$ dist $\left\{p_{n}, H_{n+1}^{\infty}\right\}$. By Lemma $3.1 u_{0}$ is a scalar multiple of a Blaschke product of $\leqq n$ factors. By Lemma 3.1 there is only one scalar multiple of a Blaschke product of $\leqq n$ factors having the form $p_{n}+$ $g, g \in H_{n+1}^{\infty}$. Consequently $u=u_{0}$ so $\operatorname{dist}\left(p_{n}, H_{n+1}^{\infty}\right)=\|f\|=M$ and it follows that $\operatorname{dist}\left(p_{n}, A_{n+1}\right)=M$, a contradiction.

Conversely, let $\sum_{i=0}^{\infty} a_{i} z^{i}=u(z)(z \in \Delta)$ where $u \in H^{\infty},\|u\| \leqq M$. If $\|u\|<M$ then given any $n \in N$ we have $M>\|u\| \geqq \operatorname{dist}\left(p_{n}, A_{n+1}\right)$. Suppose that $\|u\|=M$ and that $u / M$ is not a finite Blaschke product. Assume that $\operatorname{dist}\left(p_{n}, A_{n+1}\right) \geqq M$ for some $n \in N$. It follows that dist $\left(p_{n}, H_{n+1}^{\infty}\right) \geqq M$ and since $\|u\|=M$ we have dist $\left(p_{n}, H_{n+1}^{\infty}\right)=$ $M$. By Lemma 3.1 there is a unique function $h=p_{n}+g, g \in H_{n+1}^{\infty}$ such that $\|h\|=M$. Since $u$ has this property, $h=u$. Also, $h / M$ is a finite Blaschke product and so is $u / M$, a contradiction. 
Next we present another application of Theorem 2.3.

CoRollary 3.4. Let $z_{1}, z_{2}, \cdots, z_{k} \in \Delta$ and let $n_{1}, n_{2}, \cdots, n_{k} \in N$. Assume that $a_{i}^{j} \in X\left(1 \leqq j \leqq n_{i} ; 1 \leqq i \leqq k\right)$ where $X$ is a complex Banach space. Put $M_{0}=\inf \left\{\|g\|: g \in A(X), g^{(j-1)}\left(z_{i}\right)=a_{i}^{j}\left(1 \leqq j \leqq n_{i}\right.\right.$; $1 \leqq i \leqq k$ ) and let $F \subset T$ be a nonempty closed set of Lebesgue measure 0. Assume that $M>M_{0}$. Then given any $f \in C(F, X)$, $\|f\| \leqq M$ there is some $g \in A(X)$ which extends $f$ and satisfies $\|g\| \leqq$ $M, g^{(j-1)}\left(z_{i}\right)=a_{i}^{j}\left(1 \leqq j \leqq n_{i} ; 1 \leqq i \leqq k\right)$. This is false for $M=M_{0}$ if $M_{0} \neq 0$.

Proof. Let $L$ be a subspace of $A$ of those functions which have zero of order at least $n_{k}$ at $z_{k}(1 \leqq k \leqq n)$. Put

$$
\phi(z)=\prod_{i=1}^{k}\left(\frac{z-z_{i}}{1-\bar{z}_{i} z}\right)^{n_{i}} \quad(z \in \bar{J}) .
$$

Then $\phi \in A,|\phi(t)| \equiv 1(t \in T)$. Clearly $\mu \in L^{\perp}$ iff $\phi \mu \in A^{\perp}$. Since $A$ is a Riesz subspace of $C(T)$ and since the Lebesgue measure $m$ on $T$ is $A^{\perp}$-absolutely continuous it follows that $L$ is a Riesz subspace of $C(T)$ and that $m$ is $L^{\perp}$-absolutely continuous. Now follow the proof of Theorem 1.1.

If $\phi$ is a finite Blaschke product then $B=\phi A$ is a closed ideal in $A$ whose hull misses $T$ and if $F \subset T$ is a closed set of measure 0 then using [17, Theorem 20.12] instead of Lemma 2.1 one can prove the assertion of Lemma 2.2 for $K=\bar{\Delta}$ and consequently also the second paragraph of Theorem 2.3 for $K=\bar{\Delta}$. This enables us to prove the following partial generalization of Corollary 3.4 which generalizes and sharpens [7, Theorem p. 284-285].

Corollary 3.5. Let $z_{1}, z_{2}, \cdots, z_{k} \in \Delta$ and let $n_{1}, n_{2}, \cdots, n_{k} \in N$. Assume that $a_{i}^{j} \in X\left(1 \leqq j \leqq n_{i} ; 1 \leqq i \leqq k\right)$ where $X$ is a complex Banach space. Let $p$ be a positive continuous function on $\bar{\Delta}$ such that $\|h(z)\|<p(z)(z \in \bar{\Delta})$ for some $h \in A(X)$ which satisfies $h^{(j-1)}\left(z_{i}\right)=$ $a_{i}^{j}\left(1 \leqq j \leqq n_{i} ; 1 \leqq i \leqq k\right)$. Suppose that $F \subset T$ is a closed set of Lebesgue measure 0 and let $f \in C(F, X)$ satisfy $\|f(s)\| \leqq p(s)(s \in F)$. Then there is some $g \in A(X)$, extending $f$ and satisfying $\|g(z)\|<$ $p(z)(z \in \bar{A}-F), g^{(j-1)}\left(z_{i}\right)=a_{i}^{j}\left(1 \leqq j \leqq n_{i} ; 1 \leqq i \leqq k\right)$.

Proof. Put $B=\phi A$ where $\phi$ is defined by (11) and define $\Phi(z)=$ $\{x \in X:\|h(z)+x\|<p(z)\}(z \in \bar{A})$. Clearly $K=\bar{\Delta}, \Phi, f$ and $g=0$ satisfy the assumptions in the second paragraph of Theorem 2.3 and the assertion follows.

4. Questions and remarks. To prove the existence of Rudin- 
Carleson extensions with prescribed Fourier coefficients or some related properties the basic fact we needed about a subspace $B$ of $A$ was that

given any closed set $F \subset T$ of Lebesgue measure 0 and any $f \in C(F)$ and any positive $p \in C(T)$ satisfying $|f(s)|<$ $p(s)(s \in F)$ there is some $\tilde{f} \in B$ extending $f$ and satisfying $|\tilde{f}(z)|<p(z)(z \in T)$.

If $B$ is a Riesz subspace of $C(T)$ then $B$ satisfies (12) by Lemma 2.1. It is interesting to observe that the converse is also true so that (12) in fact characterizes the Riesz subspaces of $C(T)$. To see this, let $F \subset T$ be any closed set of Lebesgue measure 0 . It is easy to construct a positive function $p \in C(T)$ such that $p(F)=\{1\}, p(z)<$ $1(z \in T-F)$. Assume that a closed subspace $B \subset C(T)$ satisfies (12). Then it satisfies (11) with $<$ signs replaced by $\leqq[16]$. Consequently there exists a sequence $f_{n} \in B$ such that for each $n$

$$
f_{n}(F)=\{1\},\left|f_{n}(z)\right|<p^{n}(z)(z \in T-F) .
$$

If $\mu \in B^{\perp}$ then $\int_{T} f_{n} d \mu=0$ for all $n$ so by the Lebesgue dominated convergence theorem (13) implies that $\mu(F)=0$. Since $F$ was arbitrary it follows by regularity that $\mu$ is absolutely continuous [17].

We do not know whether one can characterize the Riesz subspaces of $C(T)$ by some weaker extension properties.

Note that there are closed subspaces $B \subset A$ satisfying $B \mid F=$ $C(F)$ for every closed set $F \subset T$ of Lebesgue measure 0 , which are not Riesz subspaces of $C(T) . \quad B=\{f \in A: f(0)=r f(1)\}$ [7] is an example if $r \neq 0$. However, any closed ideal $B \subset A$ satisfying $B \mid F=$ $C(F)$ for every closed set $F \subset T$ of measure 0 is necessarily a Riesz subspace of $C(T)$. For, its hull misses $T$ so $B$ satisfies (12) by [17, Theorem 20.12].

No characterization of Rudin sets seems to be known. Also, the author knows of no description of the Riesz subspaces of $C(T)$ contained in $A$.

The argument used in the proof of Lemma 2.5 is an extension of a classical argument [6, p. 133] which relates the properties of extremal functions with the fact that equality sign holds in Hölder inequality. We have seen that in order to prove that the extremal functions have constant absolute value on $T$ it is sufficient that the Lebesgue measure $m$ on $T$ is $B^{\perp}$-absolutely continuous. We ask whether the latter condition is also necessary. Also, we know of no description of closed subspaces $B \subset C(T)$ with the property that 
$m$ is $B^{\perp}$-absolutely continuous.

ACKNOWLEDGMENT. The author wishes to express his thanks to Professors I. Glicksberg, D. Marshall, V. Nestoridis and E. L. Stout for some helpful and stimulating discussions.

\section{REFERENCES}

1. E. Bishop, A general Rudin-Carleson theorem, Proc. Amer. Math. Soc., 13 (1962), 140-143.

2. J. Diestel and J. J. Uhl Jr., Vector measures, Math. Surveys, 15 Amer. Math. Soc., (1977).

3. N. Dinculeanu, Vector Measures, Pergamon Press, 1967.

4. R. E. Dressler and L. Pigno, Rosenthal sets and Riesz sets, Duke Math. J., 41 (1974), 675-677.

5. - Une remarque sur les ensembles de Rosenthal et Riesz, C. R. Acad. Sci. Paris, 280 (1975), A1281-A1282.

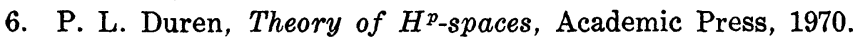

7. T. W. Gamelin, Restrictions of subspaces of $C(X)$, Trans. Amer. Math. Soc., 112 (1964), 278-286.

8. I. Glicksberg, Recent results on function algebras, Reg. Conf. Ser. in Math., Amer. Math. Soc., (1972).

9. J. Globevnik, Analytic extensions of vector-valued functions, Pacific J. Math., 63 (1976), 389-395.

10. Analytic extensions and selections, Trans. Amer. Math. Soc. 254 (1974), 171-177.

11. E. Hille and R. S. Phillips, Functional analysis and semi-groups, Amer. Math.

Soc. Colloq. Publ., 31 (1957).

12. K. Hoffman, Banach Spaces of Analytic Functions, Prentice Hall, 1962.

13. D. M. Oberlin, Interpolation and vector-valued functions, J. Functional Analysis, 15 (1974), 428-439.

14. W. Rudin, Trigonometric series with gaps, J. Math. Mech., 9 (1960), 203-227.

15. R. Ryan, Boundary values of analytic vector-valued functions, Indag. Math., 24 (1962), 558-572.

16. Z. Semadeni, Simultaneous extensions and projections in spaces of continuous functions, Lecture Notes, Univ. of Aarhus, 1965.

17. E. L. Stout, The Theory of Uniform Algebras, Bogden and Quigley, 1971.

18. S. A. Vinogradov, The Banach-Rudin-Carleson theorems and the norms of embedding operators for some classes of analytic functions. Investigations in linear operators and function theory, Part 1. (Ed. by N. K. Nikolski). Consult. Bureau, New York, 1972.

Received January 30, 1979. This work was supported in part by the Boris Kidrič Fund, Ljubljana, Yugoslavia.

UNIVERSITY OF WASHINGTON

Seattle, Washington 98195

AND

UNIVERSITY OF LJUBLJANA,

LJUBlJaNa, YUgoslavia 



\section{PACIFIC JOURNAL OF MATHEMATICS}

\section{EDITORS}

DONALD BABBITT (Managing Editor)

University of Galifornia

Los Angeles, California 90024

Hugo Rossi

University of Utah

Salt Lake City, UT 84112

C. C. MOORE AND ANDREW OGG

University of California

Berkeley, CA 94720
J. DugundjI

Department of Mathematics University of Southern California Los Angeles, California 90007

R. Finn and J. Milgram Stanford University Stanford, California 94305

ASSOCIATE EDITORS

E. F. BECKENBACH

B. H. NeumanN

F. WOLF

K. YoSHIDA

\section{SUPPORTING INSTITUTIONS}

UNIVERSITY OF BRITISH COLUMBIA

UNIVERSITY OF SOUTHERN CALIFONIA

CALIFORNIA INSTITUTE OF TECHNOLOGY

UNIVERSITY OF CALIFORNIA

MONTANA STATE UNIVERSITY

STANFORD UNIVERSITY

UNIVERSITY OF HAWAII

UNIVERSITY OF NEVADA, RENO

UNIVERSITY OF TOKYO

NEW MEXICO STATE UNIVERSITY

UNIVERSITY OF UTAH

OREGON STATE UNIVERSITY

WASHINGTON STATE UNIVERSITY

UNIVERSITY OF OREGON

UNIVERSITY OF WASHINGTON 


\section{Pacific Journal of Mathematics \\ Vol. 88, No. $1 \quad$ March, 1980}

Michael James Beeson, Extensionality and choice in constructive mathematics .................................... 1

José L. Blasco Olcina, Two questions on Wallman rings.............. 29

Peter I. Booth and J. Tillotson, Monoidal closed, Cartesian closed and convenient categories of topological spaces .................. 35

Peter B. Borwein, Rational functions with positive coefficients, polynomials and uniform approximations ......................... 55

Josip Globevnik, Fourier coefficients of the Rudin-Carleson extensions . . . . 69

Jacob Kofner, Quasimetrizable spaces ...................... 81

Mark Mandelker, Resolutions on the line ..................... 91

Lynn McLinden, An analogue of Moreau's proximation theorem, with application to the nonlinear complementarity problem ............ 101

Atsushi Murase, On the uniform distribution property of certain linear algebraic groups ................................ 163

Nicholas Th. Varopoulos, Zeros of $H^{p}$ functions in several complex

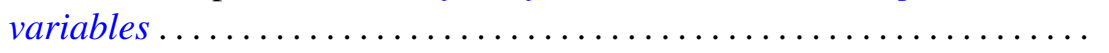

